\title{
De la collecte en milieu urbain chez les Mataco (Chaco argentin)
}

Collecting among the Mataco in the urban environment (Argentinian Chaco)

\section{François-René Picon}

\section{(2) OpenEdition}

12 Journals

Édition électronique

URL : https://journals.openedition.org/tc/5019

DOI : $10.4000 /$ tc. 5019

ISSN : 1952-420X

Éditeur

Éditions de l'EHESS

\section{Édition imprimée}

Date de publication : 30 juin 2010

Pagination : $529-543$

ISSN : 0248-6016

\section{Référence électronique}

François-René Picon, « De la collecte en milieu urbain chez les Mataco (Chaco argentin) », Techniques \& Culture [En ligne], 54-55 | 2010, mis en ligne le 30 juin 2013, consulté le 29 septembre 2022. URL : http://journals.openedition.org/tc/5019; DOl : https://doi.org/10.4000/tc.5019 


\section{DE LA COLLECTE EN MILIEU URBAIN CHEZ LES MATACO (CHACO ARGENTIN)}

in Techniques et culture 31-32, 1998: 379-395

Sollicité pour apporter sa contribution à un ouvrage rassemblant les recettes de cuisine et les manières - avant de passer à table - de préparer les aliments dans les sociétés étudiées par les ethnologues, Claude Lévi-Strauss envoya à la responsable de la publication, Jessica Kuper, la réponse suivante:

« Les tribus [indiennes d'Amérique du Sud] avec lesquelles j'ai travaillé avaient des habitudes culinaires assez grossières que l'on ne pourrait qualifier de recettes. Elles se limitaient pratiquement aux matières crues qui ne possèdent pour la plupart aucun équivalent à l'extérieur de l'Amérique tropicale. » (Kuper 1981 : 9).

Voilà donc pour l'Amérique tropicale. Pour ce qui est des chasseurs-cueilleurs, la situation n'est guère plus brillante; en effet, pour Nancy Howell Lee:

« [...] un festin Bushmen [consisterait] à marcher des kilomètres sur les dunes de Cape Cod pour [...] offrir ensuite une viande grillée coriace et criblée de sable avec deux pierres à concasser et tous les mongongos (une sorte de noix) qu'ils voulaient (c'est-àdire peu!)» (in Kuper 1981 : 9-10).

Quant à son mari, poursuit-elle, il « remarqua pensivement que son souvenir le plus tenace était la faim...».

Faire valoir que la cuisson dénature et que le cru peut constituer le suprême raffinement d'une culture ne sont pas, pour nous, des arguments vraiment convaincants; et s'il est vrai, également, que les goûts sont relatifs, il semble bien qu'il faut néanmoins reconnaître que chez les chasseurs-cueilleurs l'alimentation n'est pas réellement séduisante. Aussi 
ne sera-t-il ici question ni de recettes anciennes ni de nouvelles cuisines mais bien de ce qui les précède, c'est-à-dire de la façon dont les Indiens Mataco - chasseurs-cueilleurs d'Amérique tropicale, on l'aura compris - se procurent la matière première et les ingrédients nécessaires qui seront transformés en aliments, bref, de la façon dont les Mataco « font les courses ». On laissera donc de côté les techniques de consommation pour considérer celles qui ont trait aux modes d'acquisition, pour suivre une classification qui nous est chère.

Pour continuer à évoquer les filiations, les lignes qui suivent s'inspirent également des trop rares travaux qui, à ma connaissance du moins, traitent de ces moments anormaux dans les cycles de production que sont les époques de pénurie ou d'abondance. Dans les monographies, et a fortiori dans les ouvrages de synthèse, sont en général présentées des économies en leur point d'équilibre, en leurs moments moyens, et non ce qui s'est réellement passé durant une période précise, même s'il est évident que les années ne se répètent pas inlassablement à l'identique. Il est certain que l'on construit des systèmes et que, de ce fait, tout incite à ne pas tenir compte des écarts. Mais l'on sait bien que ces écarts sont significatifs et que c'est dans les moments extrêmes que les sociétés peuvent donner la pleine mesure de leurs capacités.

Dans le cas des Mataco, il ne s'agit pas à proprement parler de pénurie ou d'abondance mais des conséquences d'un changement de paysage dans lequel apparaît un autre système, celui de l'État caractérisé, en plus, par un certain degré de mondialisation - je veux parler des ONG et des Églises présentes sur le terrain. Il a été dit (Sahlins 1976) que le manque est une représentation, ou de façon moins approximative, je crois, que c'est le jugement énoncé par notre économie, et l'on sait comment il faut prendre cette affirmation. Ainsi, le nouveau système qui se présente manifeste en même temps la pénurie d'une ressource tout en proposant une certaine abondance. Par ailleurs, et même si ceci relève de l'évidence, l'on sait que les ressources sont relatives à leur accessibilité: il faut qu'elles soient proches et qu'un laps de temps puisse leur être consacré, au détriment parfois de l'exploitation d'autres ressources. Tout aussi évident, mais moins aisé à mesurer, il faut qu'elles soient intégrées socialement, ce qu'on peut interpréter comme la présence nécessaire d'un système de représentations accompagnant la cueillette, la chasse ou toute autre activité extractive ou prédatrice. Il y a donc en même temps des ressources proches et ignorées mais aussi des représentations parfois tellement fortes que des animaux ou des végétaux consommables doivent être laissés de côté, c'est-à-dire qu'ils sont prohibés ou tabous. Pour finir de tracer le cadre de ce texte, il pourrait fournir un exemple à ce courant qui mesure l'adaptation d'une économie à l'environnement (optimal foraging theory ${ }^{1}$ ) avec, il est vrai, l'appareil quantitatif en moins et une situation pour le moins non traditionnelle. Mais ce sont là des réserves qui ne disqualifient pas nécessairement cette illustration.

Grâce à la présence de la ville et à la société dominante, de nouvelles ressources sont à portée de main des Mataco. Exploiter ce nouveau milieu est un processus en cours depuis peu, par définition incomplet et inachevé, et qui semble - au risque d'y voir ce qui n'y est peut-être pas - comme à l'essai. La collecte urbaine pourrait évidemment être considérée comme de la mendicité ou une clochardisation des indigènes, et ce sont là des images qui nous sont immédiatement proposées. Selon le choix que l'on fait, les échos suscités sont évidemment opposés mais il contient et implique surtout des conceptions et des analyses tout à fait différentes d'une même réalité, au-delà d'une seule inclination de l'observateur pour le pessimisme ou l'optimisme. Ce que veut suggérer, en plus, le titre choisi est que la situation n'est pas inexorablement sans issue, qu'elle peut être réversible 
et qu'il s'agit de trouver les éléments qui permettraient à la fois de mieux comprendre le phénomène mais aussi, et pourquoi pas, de l'empêcher de glisser vers ce qui donnerait raison aux pessimistes. Et, à cet égard, il est tout à fait essentiel de regarder du côté des représentations, après en avoir postulé l'existence, d'essayer de les entrevoir et, au mieux, de les cerner, qu'elles concernent directement cette collecte, qu'elles se situent à un niveau beaucoup plus global ou, qu'enfin, elles soient à la jonction des deux mondes en présence.

\section{Le Chaco: aperçu ethnographique}

Las Lomitas ${ }^{2}$ - c'est le « milieu urbain » du titre - est une petite ville d'environ 8000 habitants où se termine la portion asphaltée de la route N. 81 qui vient de Formosa, capitale de la province du même nom, située à $300 \mathrm{~km}$ à l'est. Petite ville, Las Lomitas est cependant bien connue des Argentins, non pas à cause des communautés indiennes de ses environs, mais parce que l'actuel président argentin y connut l'exil pendant la dictature militaire. En ligne droite comme la route qui est faite de terre cette fois et « intransitable » quand il pleut, $400 \mathrm{~km}$ plus à l'ouest, on arrive à la frontière bolivienne d'où l'on aperçoit les Andes. Tout au long de cette N. 81, et ce bien avant Las Lomitas, s'échelonnent des villes et aussi des « communautés » indigènes, selon le terme consacré, Toba, Pilaga et surtout Mataco. Elles sont installées sur des terres qui sont, depuis 1985 environ, en propriété collective puisqu'il est d'usage de considérer que les indigènes vivent en communautés régies par les règles du collectivisme. Si ce que nous entendons par « propriété collective » approche le mieux ce qu'est la réalité indigène, elle pose cependant quelques problèmes. Que pour l'État, la société nationale ou les ONG, existe une telle propriété, on ne peut que s'en féliciter. Mais lorsque les organismes (officiels ou privés) prennent des mesures d'aide, d'assistance ou de développement et distribuent des biens, ce collectivisme attribué provoque des incompréhensions, des heurts et bien souvent l'échec des projets tels qu'ils ont été conçus. Il va sans dire que, à l'intérieur et du point de vue des communautés, ce collectivisme rêvé, souhaité, pensé et imposé de l'extérieur n'implique pas nécessairement l'absence de retombées matérielles qui se produisent, après un temps, selon des modalités « traditionnelles ». Mais là n'est pas le sujet; encore que cela n'ait pas rien à voir... comme les $300 \mathrm{~km}$ d'asphalte entre Formosa et Las Lomitas.

Il existe, à propos du Chaco, d'excellentes et très vivantes descriptions dans les textes de voyageurs et d'explorateurs comme Thouar (1891), par exemple, qui, plus chanceux que Crévaux, Ibarreta ou même Boggiani, en est revenu vivant. N'oublions pas, non plus, que Nordenskiold (1912), Métraux (1946) et Clastres (1992) ont également écrit sur le Chaco. En Argentine, cinq provinces se partagent le Chaco - Formosa, Chaco, Salta, Santa Fé, Santiago dei Estero - et c'est là que vit la quasi-totalité de la population indigène du pays. Mais le Chaco n'est pas seulement argentin. Il est également paraguayen, bolivien et quelque peu brésilien. Où commence et où finit le Chaco? Question qui s'est traduite localement et historiquement par: à qui est le Chaco?

Du côté argentin, la frontière avec le Paraguay suit le cours du fleuve Pilcomayo et celle avec la Bolivie ne provoque pas de désaccords fondamentaux. En revanche, entre le Paraguay et la Bolivie, la délimitation est si floue qu'une guerre semble inévitable pour 


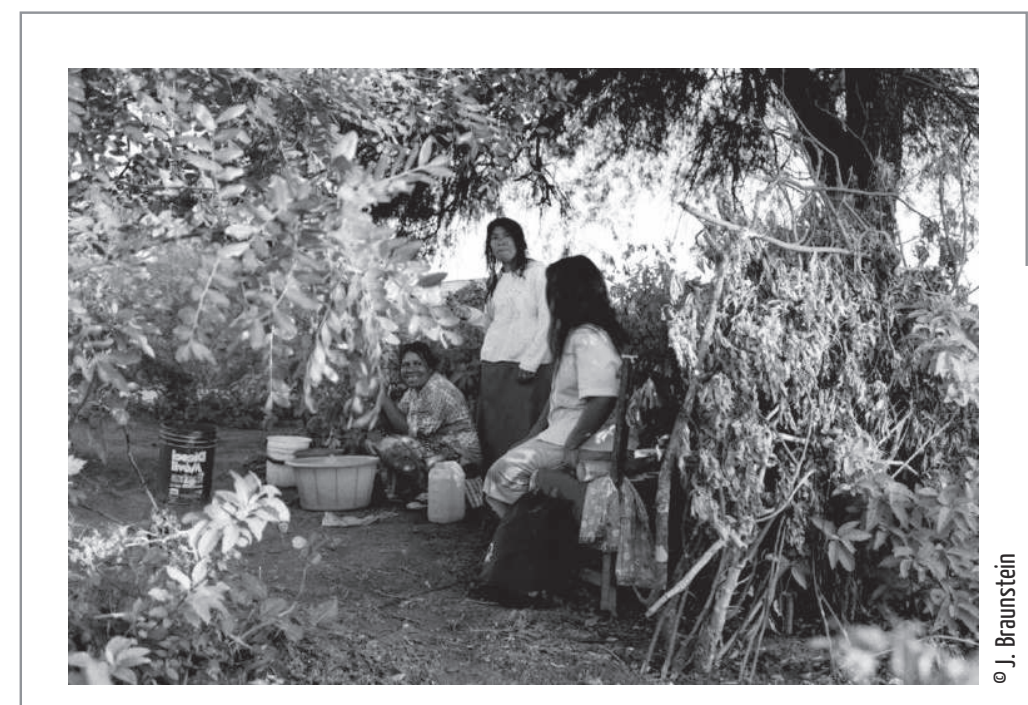

\section{Faubourgs indigènes de Las Lomitas}

Un après-midi. Femme Mataco en visite.

Conversation autour des tâches ménagères.

la préciser. C'est l'époque où, pour les deux pays, il devient impératif de s'étendre, vers l'ouest pour l'un et vers l'est pour l'autre, et le Chaco constitue donc un enjeu évident. À l'issue de la guerre du Chaco (1932-1935), la frontière est fixée et les espaces nationaux peuvent enfin s'organiser: de grandes haciendas surgissent, quelques terres sont mises en culture mais ce sont surtout les bovins qui prennent possession des terres chaqueñas. Pour les Indiens du Chaco, l'histoire récente est celle de la perte de leur espace, mais l'histoire immédiate et celle qui est en train de se faire semblent bien animées d'un mouvement inverse, beaucoup plus lent, il va de soi, car les obstacles sont multiples. Il n'en demeure pas moins que le processus de « récupération », selon le terme consacré, est en marche et, pour cette raison, il est aujourd'hui impossible, et surtout impensable, de ne pas dire quelques mots de ces questions très concrètes qui sont au centre des préoccupations vitales des indigènes et ce, même si l'on s'intéresse à des problèmes très abstraits. Il y a aujourd'hui, dans le Chaco argentin, environ 120000 indigènes $^{3}$. Les Mataco (ou Wichi) sont 44000 et vivent également en Bolivie. L'on s'accorde à reconnaître, dans les sociétés du Chaco, une assez grande homogénéité culturelle; elles ont « un air de famille ", écrit Clastres mais il précise plus loin que « chaque tribu anime ce contenu commun d'un esprit ou d'un style particulier» $\left(1992: 3\right.$ et 4) ${ }^{4}$. En la situant vers 1930, on pourrait esquisser une description ethnographique des sociétés du Chaco de la façon suivante: campements ou villages, huttes ou maisons, comme l'on voudra, ne sont pas des lieux de vie permanents. Il n'y a pas de lignages mais des parentèles où la résidence commune est le principe organisateur. On est d'un lieu et d'un groupe local. L'exogamie est de règle, que ce soit hors de la parentèle, du village et même de sa société ou tribu, linguistiquement définies. Parfois, du point de vue féminin, cette exogamie est quelque peu forcée: lors des fréquents raids intertribaux, on enlève femmes et enfants, on prend le bétail et on essaie de s'assurer un ou deux scalps de guerriers - reproduction, subsistance 
et prestige, on a là, par conséquent, un butin « total ». Sociétés guerrières donc - et ce trait paraît bien hors de doute - avec, bien sûr, des ennemis héréditaires mais aussi des alliés et des moments de paix. Une réserve, toutefois, à propos des Mataco qui viennent rompre quelque peu cette homogénéité: il semble qu'ils épousaient plus à l'intérieur du groupe et se battaient moins en dehors. Mais il s'agit de différences de degré et non d'opposition tranchée. Si l'on ne considère que l'aspect matériel, ces guerres avaient le plus souvent pour origine une lutte pour les lieux de pêche mais également pour ceux de ressources particulières.

\section{Économie et alimentation}

Les Chaqueños vivent essentiellement de chasse, de cueillette et de pêche, et les deux dernières activités comportent deux pôles saisonniers intenses impliquant des déplacements. On quitte le village pour aller vers les lieux de pêche d'avril à juillet, et pour la collecte (qui est presque une récolte) des fruits de l'algarrobo (Prosopis alba) de novembre à février, toutes deux époques de grande abondance et, en été, de fêtes et de beuveries qui rassemblent, autour de la bière d'algarrobo, de grandes quantités de gens. Le reste de l'année, l'hydromel permet aux fêtes ou occasions rituelles de se dérouler comme il se doit. En plus de la collecte ordinaire de racines, de fruits et de miel - le miel non fermenté est abondamment consommé - la chasse a une place importante et celle du nandou ou autruche américaine (Rhea americana) est particulièrement valorisée. Si les Chaqueños sont donc chasseurs-cueilleurs, ils sont aussi agriculteurs et plus éleveurs qu'on a coutume de le dire. Mais ce sont là quand même des activités mineures, complémentaires, que l'on peut considérer comme utiles pour remédier à la rareté éventuelle des ressources habituelles. Il reste qu'on peut se demander si des ressources ou activités complémentaires peuvent être qualifiées de mineures; ce sont en fait peutêtre les plus essentielles.

C'est un lieu commun que de préciser qu'il ne faut pas confondre ignorance d'une technique et sa non-pratique - ne serait-ce que parce que l'observateur ne peut pas tout voir et qu'il ne regarde que pendant un certain temps. Lorsqu'on oppose chasse, cueillette et pêche à agriculture et élevage, on ne saurait, non plus, invoquer une quelconque ignorance mais on doit, évidemment, considérer les rythmes et contraintes propres à chaque « ensemble». On ne peut pas tout faire et notamment récolter alors que commence le temps de l'algarrobo - dont l'importance alimentaire mais aussi sociale a été soulignée car tout pousse, fleurit et mûrit en même temps! Cette contradiction n'est pourtant pas sans issue comme le montre l'ethnographie des peuples du monde; les groupes peuvent se scinder et exploiter au mieux toutes les ressources, mais on sait aussi que celles qui sont abondantes et ponctuelles exercent de très fortes contraintes à différents niveaux. Dans le Chaco, c'est tout le groupe qui se déplace et, s'il y a de grands rassemblements, la division de la plus petite unité sociale - le campement - n'est pas concevable. De toute évidence, il faut opérer un choix et ce qui semble bien, en partie, le guider est l'attraction (ou la contrainte?) de ces moments d'abondance «sociale » tout autant qu'économique.

Il en va de même pour l'élevage: que faire de trop d'animaux lors des mouvements saisonniers quand et puisque tout le monde se déplace? Ce qui frappait les observateurs 
du début du siècle et ce qui est toujours manifeste et, qui sait, peut-être encore plus prononcé aujourd'hui, c'est le comportement « amateur », ou, pour lever toute ambiguité, dilettante des Chaqueños face à ces deux activités sur lesquelles missionnaires et développeurs font porter tous leurs efforts. Ils en préfèrent évidemment d'autres, plus cohérentes avec la chasse et la cueillette ou avec le « style nomade » (Lee \& DeVore 1968 : 11), expression qui montre combien il est malaisé de définir les chasseurs-cueilleurs (Arcand 1988). On pourrait également, pour continuer à mettre en question le bien-fondé de la catégorie, mais aussi pour voir les choses sous le meilleur jour possible, considérer qu'agriculture et élevage sont en réserve, comme des savoirs de survie qu'il est impératif de conserver en mémoire et de pouvoir pratiquer et activer quand il le faut ${ }^{5}$.

Enfin, pour abandonner un temps les techniques et passer aux représentations, tout un ensemble de mythes rend évidemment compte de ces activités, que ce soit de l'origine des plantes cultivées ou de l'usage des plantes et ressources sauvages. Outre des esprits qu'il s'agit de se concilier avant et après la chasse, chaque espèce animale (sauvage) « appartient » à une figure appelée « maître des animaux ». On note enfin dans les mythes une présence occasionnelle des animaux domestiques qui occupent un tout autre registre que les animaux sauvages.

\section{Les facteurs de changement}

Cette situation « traditionnelle » s'est maintenue jusque dans les années trente, car les présences nationales dans le Chaco étaient pratiquement nulles (surtout au Paraguay) et les Indiens, on l'a dit, maîtres du terrain. Mais elle s'est, bien entendu, peu à peu modifiée et la pression sur les terres n’a cessé d'augmenter. Du côté argentin, outre les expéditions qui ont traversé le Chaco avec des fortunes diverses, il faut rappeler la construction de la ligne de fortins qui favorisait certes une mission civilisatrice mais protégeait surtout l'exploitation du quebracho (Schinopsis balancea ou lorenzi) riche en tanin, et une voie ferrée permettant l'acheminement des troncs vers les usines de traitement situées aux frontières sud et est du Chaco. Avec un apogée vers 1930, cette activité s'est bien sûr ralentie au rythme de l'épuisement des arbres.

Une autre industrie a eu un impact beaucoup plus grand sur les sociétés indigènes. Les plantations et raffineries de canne à sucre - ou ingenios - du Nord-Ouest de l'Argentine se trouvaient presque en dehors du Chaco (on y accédait en effet sans le traverser), mais elles drainaient cependant, à partir de la fin du xix $x^{e}$ siècle, une main-d'œuvre indigène considérable qui venait parfois de fort loin. Le travail effectué y était payé principalement en nature et c'est par cet intermédiaire que les biens manufacturés sont entrés chez les Chaqueños. On ne peut cependant pas avancer que ces ingenios ont bouleversé fondamentalement l'organisation sociale et le mode de vie indigènes. On pourrait même dire qu'ils ont contribué à les maintenir pour un temps car c'était, pour certains groupes, l'occasion de se retrouver pour les fêtes collectives et ce, surtout à partir du moment où le Chaco commençait à être colonisé et les mouvements saisonniers traditionnels des indigènes de plus en plus difficiles ${ }^{6}$. Vers 1940, le nombre d'ouvriers indiens a commencé à baisser et la mécanisation, en 1960, a définitivement fermé ce qui était plus qu'une source de travail. 
Lactivité missionnaire a commencé chez les Lengua (ou Enxet) en 1890 par l'installation au Paraguay d'une mission anglicane. Après des débuts difficiles en raison de la dureté du climat et de l'hostilité des Indiens, elle a pris une grande extension et le premier centre (Makthlawayia), fondé en 1907, n'était pas sans rappeler l'ambition des Réductions jésuites. Il a fermé il y a quelques années après ce que l'on pourrait appeler une prise de conscience des missionnaires. Questionnement louable mais dont les conséquences ne furent pas nécessairement positives, et l'on peut étendre cette même réserve à d'autres ordres missionnaires qui ont changé l'orientation de leur politique. Présente également en Argentine, l'Église anglicane s'est un peu mise en retrait mais elle y reste fortement implantée. Sur les neuf ordres ou églises présents dans le Chaco qui s'occupent des âmes des Indiens et trop peu de leurs terres, l'Église Évangélique Unie et les Mennonites semblent avoir aujourd'hui une certaine influence, fondée sur des moyens certains.

Enfin, confessionnelles ou laïques, les ONG viennent s'ajouter - et parfois remplacent - les missions traditionnelles. Quel que soit leur bord, elles s'occupent des terres et du développement, défendent, enseignent et aident, et le souci des âmes indiennes n'étant pas prioritaire, il est laissé aux Indiens. Eu égard à la philosophie dominante dans les ONG, on peut toutefois se demander si aider à revendiquer des droits ne va pas sans influencer les âmes, même si c'est indirectement et pour la bonne cause.

Las Lomitas donc, une gare presque désaffectée, un hôpital avec quatre médecins et des consultations où viennent les indigènes, deux ou trois plazas, quelques avenues ombragées avec une allée centrale où l'on se promène le soir... mais surtout des rues longues d'une centaine de mètres qui se coupent à angle droit, des rues non goudronnées bordées de fossés sur lesquels des planches sont jetées pour pouvoir accéder aux maisons car, lorsqu'il pleut, sable, terre et poussière se transforment en boue et les fossés conservent l'eau plus qu'ils n'en assurent l'écoulement.

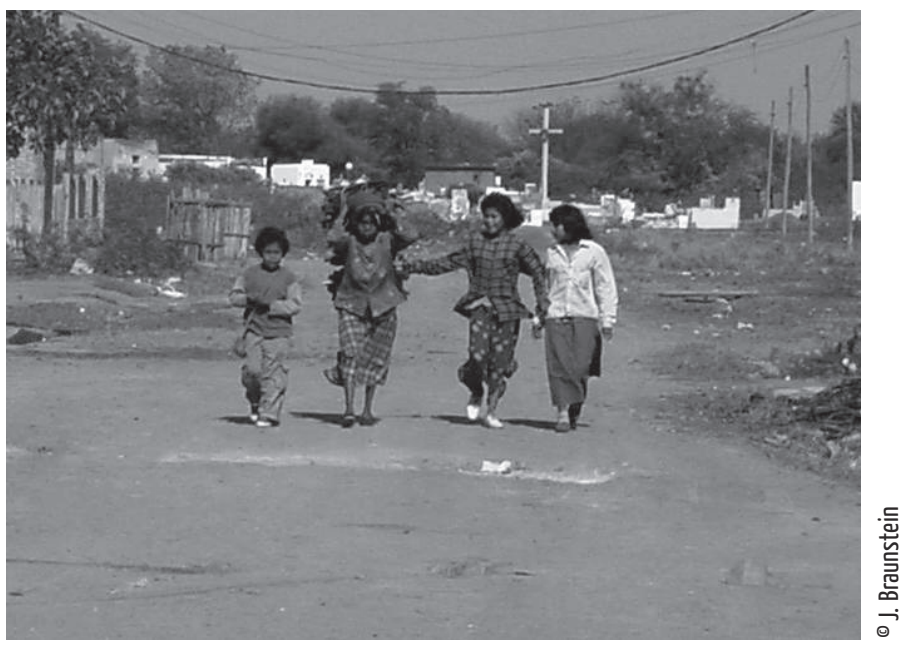

Femme et enfants Mataco en route pour Las Lomitas après avoir ramassé du bois dans les environs. 


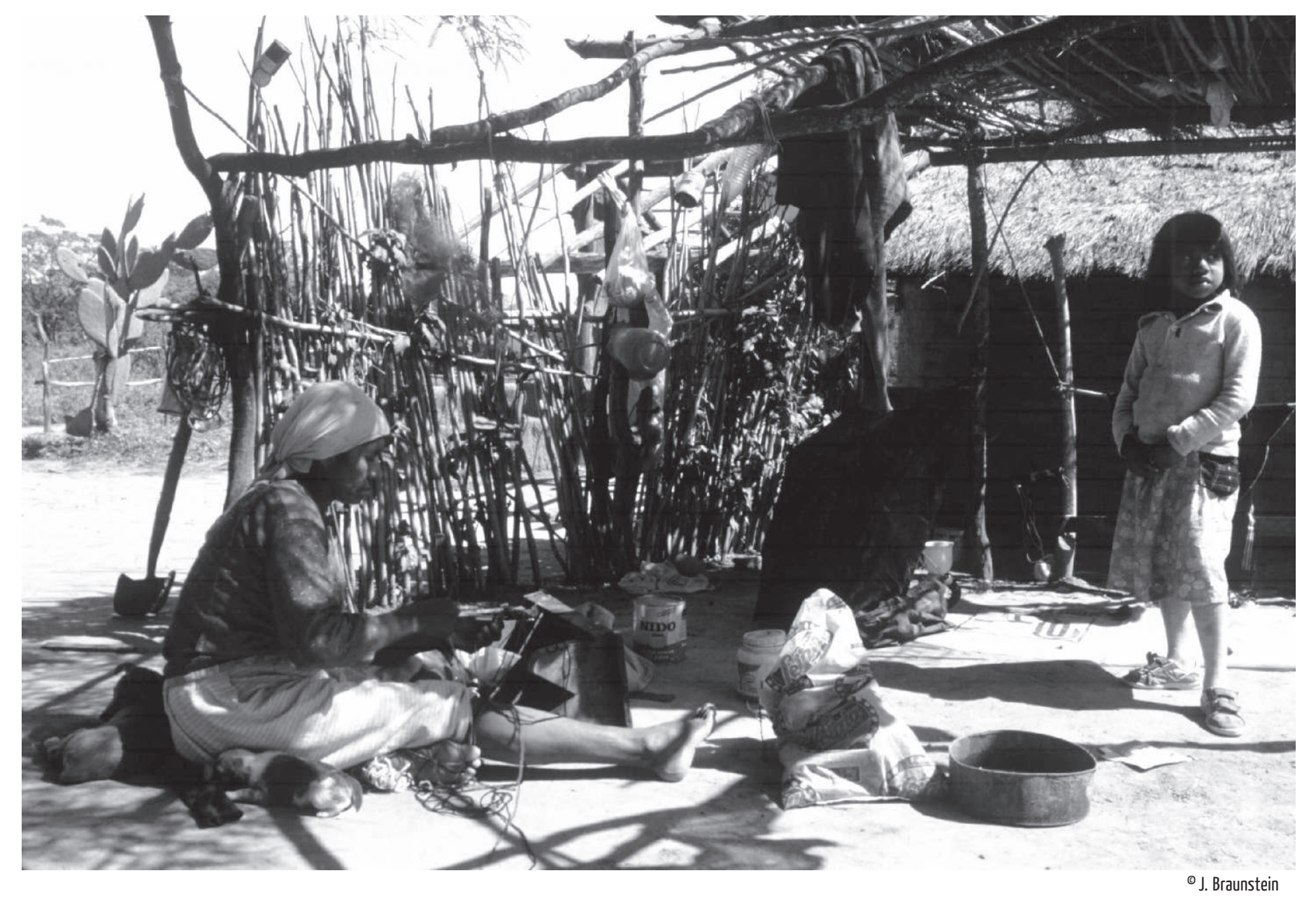

Banlieue indigène de Las Lomitas.

Femme Mataco tissant une ceinture dans la cour de son habitation (toldo ou rancho).

En arrière-plan l'espace « cultivé ».

Les abords immédiats de la ville et surtout au nord de la N. 81 ne montrent pas la même rigueur dans la disposition des maisons et l'agencement des rues; il s'agit plutôt d'un urbanisme sauvage, la population y est presque exclusivement indigène et les lots sont en propriété individuelle. En revanche, lorsqu'on s'éloigne en quelque direction que ce soit de 6 ou $7 \mathrm{~km}$, vers l'aéroport, par exemple, où n'atterrissent que des vols exceptionnels, on trouve des terres qui sont la propriété collective des communautés indigènes. On a donc, tant en zone urbaine qu'à faible distance, une population indigène relativement importante et, comme l'on peut s'y attendre, dans la majorité des cas d'étroites relations existent entre ceux qui vivent dans l'un et l'autre lieux. La faible superficie des lots urbains et individuels ne permet guère d'activités de production. Il y a cependant quelques animaux qui vaguent ça et là et l'on voit parfois de rares cultures à l'échelle d'un jardinet. Dans les lots collectifs, en revanche, l'espace ne manque pas vraiment - pour l'instant du moins -, la superficie des friches en témoigne; mais ce n'est pas pour autant que cultures ou élevage semblent, sinon intensément, du moins normalement pratiqués. 


\section{Un nouveau mode \\ d’acquisition de la nourriture}

Quelques jours après mon arrivée dans la ville, je remarquais des groupes de femmes indiennes et d'enfants marchant dans les rues et s'arrêtant parfois face à des maisons dont celle où j'habitais ${ }^{7}$. Dans la matinée, des silhouettes se tenaient à l'entrée ou passaient très lentement devant les fenêtres. Ni appel, ni coups frappés à la porte, mais simplement là, jusqu'à ce qu'on les remarque, qu'on prépare ce pour quoi ils étaient venus, qu'on ouvre la porte et que l'on donne. Tout ça se passait dans le plus complet silence, à la rigueur quelques mots étaient échangés, mais ni demande ni merci. Tous les jours, ou un jour sur deux, se répétait la même scène silencieuse. S'il est certain que l'endroit où vit un ethnologue connaît beaucoup d'allées et venues, cette «mendicité »-au début l'image était trop forte ou trop facile - s'exerçait également dans d'autres lieux, dans d'autres maisons, celles des membres des ONG, des notables mais aussi celles des gens ordinaires. On va bien sûr là où l'on vous donne, là où l'on peut s'approvisionner; et selon les maisons, qui sont autant de lieux d'acquisition, ce que l'on reçoit est différent. Enfin, pour être exact, les enfants ne demandaient pas et on venait aussi proposer quelques vanneries ou des ceintures de laine tissées qu'on vendait parfois ou, pour être plus fidèle à ce que j'ai ressenti, qu'on venait échanger contre de l'argent. Des gens des maisons, on recevait donc toutes sortes de choses, pain, farine, viande (crue ou cuite), légumes, vêtements, boissons gazeuses. Alors, peu à peu se précise une image: ce n'est pas le « donnez-moi n'importe quoi» mais plutôt le « je suis là pour ça, vous le savez bien...», ce n'est plus vraiment l'image de la mendicité, même s'il en coûte de se défaire de celle de l'Indien clochardisé.

Me retrouvant dans une communauté indigène, à environ $35 \mathrm{~km}$ à l'est de la ville et 30 minutes lorsqu'il ne pleut pas, alors que je faisais mon enquête sur l'élevage, cette collecte urbaine me revint peu à peu à l'esprit. En position de visiteur cette fois, mais de ces visiteurs qui restent, j'ai eu le sentiment de retrouver ce

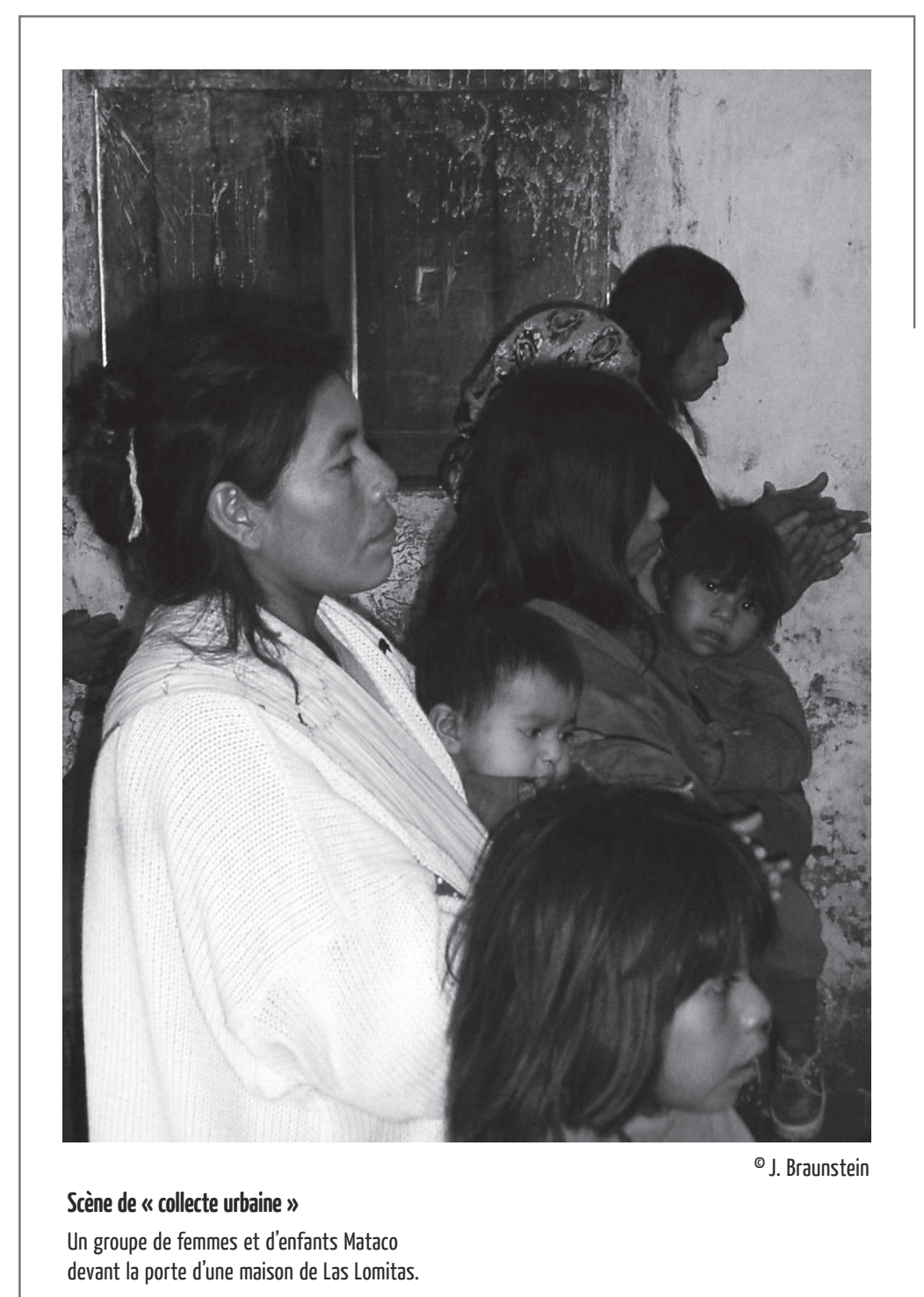


même « contrat » et ses termes. Je ne recevais aucune aumône, on m'a donné, j'ai reçu, j’ai donné (et non rendu), j'ai aussi acheté des choses, on m'en a par ailleurs donné d'autres... Il s'agissait, je crois, de ne pas laisser l'une ou l'autre inflexion - cadeau ou vente - prendre trop d'importance. Il est bien et il est sain de ne pas les séparer l'un de l'autre et de maintenir une ambiguité, celle du don, qui laisse les relations ouvertes. En ville - chez nous - comme dans la communauté - chez eux - les mêmes mécanismes, me semble-t-il, étaient à l'œuvre.

La communauté de Tres Pozos, fondée vers 1960, a vu sa population décroître au cours de ces dernières années - de trente-sept familles en 1987 à une vingtaine en 1992. Pourquoi cet exode d'un lieu où l'espace ne manque pas et d'où le fleuve Bermejo est relativement proche? Il est des phrases simples qui en disent long, ou qui sont à la jonction de plusieurs réalités et de mondes différents. "Ici, il n’y a pas de travail », ou encore « le fleuve est loin...», me disait-on. C'était en avril, début du temps de la pêche et un sentier part du village jusqu'au fleuve. Entre les nombreuses prises de mate on a consommé des fruits sauvages, du miel et du potiron, mangé un armadilla et l'ordinaire était fait de lanières de viande séchée que l'on fait bouillir longtemps; on est allé chercher de quoi refaire le toit de l'une des maisons et on a rapporté des fruits sauvages au goût d'artichaut que l'on a fait cuire sous la braise. Mais il n'y a toujours pas de travail et le fleuve Bermejo est tellement loin... Il est quand même étonnant que tout ce qui a fait l'essentiel de la vie puisse être ainsi progressivement abandonné; les choses se font toujours un peu, presque accessoirement, mais le centre (ou le sens) semble être ailleurs. Et l'on ne peut s'empêcher de s'interroger: déstructuration, anomie ou bien flexibilité? Il faudrait, je crois, se décider.

Si la description n'a été que très sommairement esquissée, c'est que, d'un côté, ce n'était pas le but de mon enquête. On a tous eu cependant l'œil attiré par des faits que l'on n'a pas voulu ou pas eu le temps d'approfondir ou, encore, qu'on a regretté, après coup, de ne pas avoir regardé de plus près. Je n'ai donc pas tous les éléments nécessaires pour construire une description qui s'articulerait, par exemple, sur les points suivants: tel groupe, composé de telles personnes passe x temps entre les maisons a, b, c, n; il obtient une certaine quantité de biens qu'il rapporte en tel endroit et qui va être partagée (si c'est le cas) entre $x, y$, et $z$ dont les relations sont de telle nature, etc. Par ailleurs, d'autres sont peut-être (cf. note 5) en train de faire cette étude avec toute la précision voulue, auquel cas l'idée générale leur revient de droit. La question qui se pose alors est de savoir si l'on peut expliquer un phénomène sans l'avoir au préalable décrit sous toutes ses coutures? Linterprétation demande-t-elle que soient épuisés tous les niveaux de description? Ou bien y a-t-il des niveaux d'interprétation - je ne fais aucune hiérarchie - qui correspondraient chacun à des niveaux de description?

Ainsi, plus que de montrer comment se fait cette collecte, il s'agit ici d'envisager une certaine catégorie de pourquoi, non pas celui de l'explication ultime, mais plutôt du « comment se fait-il qu'ils fassent cela? ». Cette question renvoie au contexte de départ, c'est-à-dire au système traditionnel mais aussi aux lieux situés en dehors de la ville - dans le cas présent la communauté de Tres Pozos. S'interroger ainsi c'est aussi, par ailleurs, tenter de caractériser le système des activités tel qu'il était pratiqué et surtout tel que les gens peuvent se le représenter - et, en ce point, le terrain est bien peu sûr! C'est donc choisir un niveau d'explication bien fragile, mais pourquoi n'y aurait-il pas de place pour le « j'ai l'impression que... », à condition toutefois, non pas de l'avouer du bout des lèvres, mais de le préciser clairement. 
Même si elle est schématiquement exposée, je ne voudrais pas donner une image fausse de cette collecte: sans être marginale, elle n'est pas générale. Certains la pratiquent, d'autres non, certaines familles ou certains individus plus que d'autres, mais il semble que ceux qui la font aient tendance à la faire régulièrement. Répondre en invoquant que ceux-là sont les plus pauvres simplifierait beaucoup trop la réalité. La pauvreté est un vieux débat en anthropologie. J'en dirais seulement qu'en situation urbaine et « rurale » beaucoup de temps est consacré à ne rien faire. Or, on l'a vu, dans le second cas les terres ne manquent pas, au sens où beaucoup sont inutilisées. Il y a de toute évidence un non-faire qui évoque avec insistance le célèbre « je préférerais ne pas le faire » de Bartleby, ce personnage de Melville, qui, on le sait, fait autre chose que ce qu'il devrait faire. Traduit en termes extérieurs cependant, ceux des ONG, par exemple, on a pauvreté et ignorance technique. Certes, je le répète, la communauté anthropologique ne fait pas de telles confusions (« nous savons bien que si vous ne faites pas, ce n'est pas parce que vous ne savez pas faire! ») mais il faut avouer que son impact sur les Indiens et leur façon de vivre est bien faible et c'est là d'ailleurs une non-ingérence qui est presque une revendication de l'anthropologie. En revanche, les ONG sont beaucoup plus écoutées, en grande partie parce qu'elles ont les moyens de se faire entendre: elles agissent et assistent les Indiens parce qu'ils sont pauvres et ne savent pas faire certaines choses. Et par conséquent, à la condition de se penser euxmêmes tels que certains les pensent, les Indiens seront aidés et leur seront enseignées des choses qu'ils savent faire et des savoirs qu'ils détiennent - à leur façon - et les résultats seront à la mesure de l'erreur de départ. Mais d'un côté, cette critique des ONG est trop facile et injuste; de l'autre, je souhaite éviter pour le moment le débat d'une anthropologie qu'appliqueraient des anthropologues! Il s'agissait seulement d'envisager la possibilité que s'il y a collecte urbaine, c'est qu'il est peut-être bon pour les Indiens de correspondre à l'image que certains ont d'eux.

Dans les dernières vingt années, les sociétés indigènes d'Amérique du Sud (tout comme celles, bien entendu, d'autres régions du monde) ont changé et ont pris un tournant; si elles doivent toujours s'ajuster, ce n'est plus à une domination mais, précisément pour s'en libérer, à une situation nouvelle, situation qui est aussi une image, et ce pour faire reconnaître leurs droits. On est en effet passé de l'imposition d'un ordre, d'une exploitation matérielle à l'imposition d'une image issue d'un imaginaire particulier où la mauvaise conscience coloniale est toujours déterminante. Et à cet égard, on peut se demander si cette nouvelle acculturation, plus subtile et moins violente, n'est pas tout aussi dangereuse. Il n'est pas certain qu'elle le soit; car si l'image peut devenir réalité ou être profondément intériorisée, elle peut être aussi adaptée et utilisée dans un double discours tenu consciemment: on peut tout à la fois paraître être et être autre - tel un acteur qui, sur scène, jouerait mais aussi se jouerait du public. Ce que l'on accorde à l'acteur, ne pourrait-on pas le reconnaître également aux sociétés indigènes et accepter qu'elles puissent supporter pour elles et dans leur intérêt politique le poids d'une double identité?

\section{Essai d'interprétation}

Du point de vue de l'observateur, à rester en ville, on reste dans la mendicité. Si, en revanche, on essaie de chercher comment les Mataco font-et-pensent cette mendicité, il faut aller vers 
le contexte de départ, c'est-à-dire le système global et tenter de le caractériser. On se trouve dans un système à dominante de chasse, pêche et cueillette - que l'on décrit et qualifie à l'aide des notions de fluidité, souplesse, diversification et flexibilité et où les individus font des choix et preuve d'opportunisme.

Serait-ce maintenant prendre trop de risques que de se demander comment les Mataco peuvent se représenter ce système? Si l'on va vers la ville, lieu du travail et où, après tout, il peut y en avoir, c'est qu'il y existe également des ressources - cette collecte et les ONG - non perçues comme du travail et qui sont représentées comme rare dans la communauté, hors de la ville.

Enfin, des points de vue de l'observateur et de l'indigène, quelle est la différence entre le pain qui vient d'une maison et le fruit d'un arbre? C'est bien sûr celle qui existe entre les représentations. Nous connaissons les nôtres; mais quelles seraient celles qui sous-tendent la collecte urbaine et le rapport aux ONG? Si par représentation, on entend mythe, il n'y en a pas, pas encore ou alors je ne les ai pas trouvés - ce serait le mythe de l'origine du pain ou des outils donnés par les ONG. Pris dans un sens plus large, la représentation n'est pas seulement quelque chose qui s'énonce mais c'est aussi ce que permet le système, la flexibilité, par exemple. Lorsqu'on considère ce qui est dit, le: « ici, il n'y a pas de travail » - et qui est énoncé en espagnol, langue du travail -, on a une explication qui est aussi une représentation, elle est empruntée aux autres et intégrée dans le système grâce à cette flexibilité, ou ce «style », pour revenir à Clastres et à Lee et DeVore ${ }^{8}$.

Ainsi peut-on pratiquer la collecte dans un monde où il y a du travail et, d'une certaine façon, cette collecte devient travail ; ou du moins n'y a-t-il pas cette absence insupportable, ce manque et donc cette pauvreté renvoyée par le miroir d'un autre système. Se contenter de peu est un autre problème; c'est plutôt la non-existence et le non-accès à une catégorie, même si elle est étrangère, qui rend la situation difficile. Il est essentiel de tout avoir à portée de main, ne serait-ce que sous forme de " possibles ». Aussi cette manière de s'approvisionner peut-elle être considérée comme une extension du « mode » chasseur-cueilleur, une prédation qui s'exerce dans un nouveau milieu que les Mataco ont, d’une certaine façon, apprivoisé, naturalisé ou cynégétisé. Bien évidemment, ces mots restent des à-peu-près qui, au mieux, décrivent une interprétation et suggèrent une image qu'il m'a été donné de voir. Puisqu'elle se fait en ville et qu'elle est productive, cette collecte n'est pas exempte de certaines des valeurs attachées au concept de travail. Elle constitue aussi, je le rappelle, un moment d'un processus: certains Mataco, pour le moment, recueillent divers biens et aliments au cours de trajets qu'ils effectuent en milieu urbain. On peut ne pas, ou ne pas pouvoir, résister au changement; on peut aussi changer en résistant, c'est-à-dire accepter de nouvelles valeurs mais, après un temps, pouvoir les remodeler. Ainsi peut-on se demander si un jour la collecte traditionnelle ne pourrait pas être perçue, à son tour, comme un travail, un autre travail ou un travail équivalent de ce fameux travail qui est absent des lieux où sont établies les communautés indigènes. Si l'anthropologie a un rôle pratique à jouer et si elle peut enseigner quelque chose aux gens dont elle tire sa matière, ce serait d'expliquer, affirmer et répéter, tant à eux-mêmes qu'à l'extérieur, que le monde indigène est équivalent au monde qui l'entoure et qu'il doit être vécu et compris comme tel. 
1. «Théorie du rendement optimal » ou encore « théorie de la prédation optimale »; bien qu'elle soit « officielle» (Hladik 1996), la première traduction me semble prendre trop de liberté avec l'expression originale.

2. Ces quelques réflexions sont issues d'une enquête (qui avait un tout autre but) menée au cours des mois d'avril-mai 1995 dans la ville et la région de Las Lomitas, province de Formosa, dans le Nord de l'Argentine.

3. Selon une estimation communiquée par José Braunstein.

4. Le Chaco n'est pas une région d'Amérique aussi célèbre que peuvent l'être l'Amazonie ou les Andes. Les informations cependant ne manquent pas. On trouvera donc la plupart des titres dans un article de Sterpin (1993) concernant les Nivacle (ou Chulupi ou encore Ashluslay). Il faut ajouter les ouvrages d'Alvarsson (1988) sur les Mataco de Bolivie, de Schindler (1983), sur l'histoire des Indiens équestres, du missionnaire anglican Grubb (1993) - ainsi que deux articles de Kidd (1994 et 1995) - sur les Lengua (ou Enxet) du Paraguay et enfin celui de Stunnenberg (1993) pour les données les plus récentes sur les sociétés indigènes face au problème foncier au Paraguay et en Argentine.

5. Non loin du Chaco, de l'autre côté du fleuve Paraguay et dans un autre environnement, je ne peux m'empêcher de penser à ce propos aux Aché (Guayaki), paradigme, à bien des égards, des chasseurs-cueilleurs même s'ils ont, auparavant, pratiqué l'agriculture et l'ont « oubliée » par la suite. Puisqu'ils n'ont plus guère de forêt où chasser, ils sont installés aujourd'hui dans des villages autour desquels ils cultivent maïs, manioc, bananes, etc. ; leur compétence ne peut être seulement expliquée par l'assistance très sporadique qui leur est apportée.

6. L'attraction exercée par les ingenios a commencé bien avant que ne soit réellement menacé le nomadisme saisonnier des Chaqueiios. Elle s'explique avant tout parce qu'y travailler permettait d'obtenir les objets des Blancs mais on ne peut oublier le « goût» pour les grands rassemblements. Et s'il est certain que l'ingenio est moins traditionnel que l'algarrobo, il reste que l'on peut y voir à l'œuvre le même « style » social, cette fois.

7. Je voudrais ici préciser les conditions - dont on ne parle pas assez et qui sont parfois déterminantes dans lesquelles j'ai observé cette collecte. Je ne l'ai pas du tout découverte et une bonne partie du développement que j'en ferai ne m'appartient pas. À Las Lomitas, j'ai donc vu des Indiens allant et venant dans les rues et s'arrêtant devant des maisons. J'ai posé des questions et on m'a expliqué. "On », ce sont mes hôtes, J. et A. Braunstein, un couple d'anthropologues du CONICET qui résident dans cette ville depuis des années et m'ont très gentiment reçu et hébergé. Grâce à eux, j'ai pu mener l'enquête que je souhaitais, celle sur tout autre chose que la collecte urbaine. Mais il est certain que nous en avons discuté. Il était même question d'en faire une étude détaillée et peut-être est-ce en train de se faire. Reste que je ne me souviens pas jusqu'où sont allées nos discussions. Je sais, en revanche, que qualifier cette pratique de «mendicité » simplifiait et faussait la réalité, du moins écartait ce qui pouvait être une explication plus globale. Aussi aimerais-je partager avec eux l'intérêt que peuvent susciter ces réflexions mais être seul responsable si elles s'avèrent triviales ou par trop spéculatives.

8. Que l'on fasse appel comme ici au «style » et à l'économie ou à «l'identité ethnique » et aux «valeurs morales »-autonomie, partage et égalitarisme qui les sous-tendent-, comme le fait Renshaw (1988) pour rendre compte de la préférence des chaqueños pour le travail salarié, la question reste bien la même: celle de la persistance de certains traits en situation de contact prononcé ainsi que de la possibilité de leur traduction d'un système à l'autre. 


\section{RÉFÉRENCES}

Alvarsson, J. A. 1988 The Mataco of the Gran Chaco. Uppsala: University of Uppsala.

Arcand, B. 1988 Il n'y a jamais eu de société de chasseurs-cueilleurs, Anthropologie et Société 12 (1): 39-58.

Clastres, P. 1992 Mythologie des Indiens Chulupi. Paris: Éditions de l'EPHE.

Grubb, W.B. 1993 Un Pueblo desconocido en tierra desconocida. Asunción: Biblioteca Paraguaya de Antropología.

Hladik, C. M. \& al. 1996 L'Alimentation en forêt tropicale, vol. II. Paris: UNESCO.

Kidd, S. 1994 The Enxet of Paraguay and their struggle to regain their land, Indigenous Affairs 1.

— 1995 Land, politics and benevolent shamanism: the Enxet Indians in a Democratic Paraguay, Journal of Latin American Studies 27 (1): 43-75.

Kuper. J. (dir.) 1981 La Cuisine des ethnologues. Paris: Berger Levrault.

Lee, R. B. \& I. Devore (dir.) 1968 Man the hunter. Chicago: Aldine.

Metraux, A. 1946 Ethnography of the Gran Chaco. In J. H. Steward (dir.) Handbook of South American Indians, vol. 1. Washington: Smithsonian Institution: 197-370.

Nordenskiöld, E. 1912 La vie des Indiens dans le Chaco, Revue de Géographie.

Renshaw, J. 1988 Property, resources and equality among the Indians of the Paraguayan Chaco, Man, n.s. $23: 334-352$

Sahlins, M. 1976 Âge de pierre, âge d'abondance. Paris: Gallimard.

Schindler, H. 1983 Die reiterstämme der Gran Chaco. Berlin: D. Reiner.

Sterpin, A. 1993 La Chasse aux scalps chez les Nivacle du Gran Chaco ,Journal de la Société des Américanistes LXXIX: 33-66.

Stunnenberg, P. 1993 Entitled to land. Saarbrück: Verlag Breitenbach.

Thouar, A. 1891 Explorations dans l'Amérique du Sud. Paris: Hachette. 


\section{RÉSUMÉ}

De la collecte en milieu urbain chez les Mataco (Chaco argentin). Ce texte présente les techniques d'acquisition de certains aliments, pratiquées dans un contexte urbain par des Indiens Mataco vivant au voisinage de la ville de Las Lomitas, dans le Gran Chaco argentin. On insiste tout d'abord sur le fait qu'il s'agit d'un phénomène récent et d'un processus en cours. Puis on tente de dépasser l'image de la mendicité habituellement associée à l'urbanisation des minorités pour présenter enfin une interprétation de cette collecte urbaine dans laquelle les représentations émanant de la société nationale se mêlent étroitement avec celles qui sous-tendent une économie essentiellement fondée sur la chasse, la pêche et la cueillette.

\section{ABSTRACT}

Collecting among the Mataco in the urban environment (Argentinian Chaco). This article presents the acquisition techniques for certain foodstuffs used in an urban context by the Mataco Indians living near the town of Las Lomitas in the Argentinian Gran Chaco. Emphasis is placed on the fact that the phenomenon is recent and the process is still evolving. The author goes beyond the image of begging usually associated with the urbanization of minorities to present at last an interpretation of this urban collecting in which the representations issuing from national are intimately mixed with those underlying an economy based on hunting, fishing and collecting.

\section{MOTS CLÉS}

Gran Chaco, chasseurs-cueilleurs, techniques d'acquisition, urbanisation, Indiens Mataco.

\section{KEYWORDS}

Gran Chaco, hunters-gatherers, acquisition techniques, urbanization, Mataco Indians.

\section{NOTES ET RÉFÉRENCES DE L'INTRODUCTION}

1. Encore une fois, ma gratitude à José Braunstein, dont les photographies donnent une nouvelle dimension au texte initial. Photo d'ouverture : Las Lomitas. Femme Mataco se dirigeant vers le centre-ville pour livrer (échanger ? vendre ?) du bois. 